Objectives Several epidemiologic studies indicate an increased risk of lung cancer among cooks but it is not known whether this is caused by cigarette smoking or by occupational exposure to carcinogens. Emissions from high-temperature frying have been classified by the IARC as probably carcinogenic to humans.

Methods We used data from the SYNERGY project with pooled information on lifetime work histories and tobacco smoking from 13176 lung cancer cases and 16129 controls from 11 case-control studies in Europe and Canada. There were 704 persons (405 men, 299 women) who had ever worked as a cook or kitchen worker (based on ISCO-68), among them 340 cases and 364 controls. ORs and 95\% CIs were estimated by unconditional logistic regression, adjusted for study, age, sex, smoking, and ever employment in an occupation with established lung cancer risk.

Results Occupation as a cook or kitchen worker was associated with an increased lung cancer risk before (OR 1.20, $95 \%$ CI 1.03 to 1.40 ) but not after (OR 1.01, 95\% CI 0.86 to 1.20) controlling for smoking habits. There was no significant exposure-response relationship in terms of work duration, and no significant heterogeneity in lung cancer risk among cooks across studies. It was not possible to separate cooks from other kitchen workers.

Conclusions Working as a cook or kitchen worker was not associated with an increased risk of lung cancer. However, the possible risk by cooking fumes cannot be ruled out. Misclassification of exposure may have biased our results towards the null.

\section{P163 LUNG CANCER RISK AMONG COOKS AND KITCHEN WORKERS IN A POOLED ANALYSIS OF CASE-CONTROL STUDIES IN EUROPE AND CANADA}

Carolina Bigert, ${ }^{1}$ Ann Olsson, ${ }^{2}$ Per Gustavsson, ${ }^{1}$ Thomas Brüning, ${ }^{3}$ Benjamin Kendzia, ${ }^{3}$ Beate Pesch, ${ }^{3}$ Karl-Heinz Jöckel, ${ }^{4}$ Hermann Pohlabeln, ${ }^{5}$ Wolfgang Ahrens, ${ }^{5}$ Isabelle Gross, ${ }^{3}$ Irene Brüske, ${ }^{6}$ Heinz-Erich Wichmann, ${ }^{6}$ Franco Merletti, ${ }^{7}$ Dario Mirabelli, ${ }^{7}$ Lorenzo Richiardi, ${ }^{7}$ David Zaridze, ${ }^{8}$ Adrian Cassidy, ${ }^{9}$ Neonila SzeszeniaDabrowska, ${ }^{10}$ Peter Rudnai, ${ }^{11}$ Jolanta Lissowska, ${ }^{12}$ Isabelle Stücker, ${ }^{13}$ Eleonora Fabianova, ${ }^{14}$ Rodica Stanescu Dumitru, ${ }^{15}$ Vladimir Bencko, ${ }^{16}$ Lenka Foretova, ${ }^{17}$ Vladimir Janout, ${ }^{18}$ Jack Siemiatycki, ${ }^{19}$ Maria Teresa Landi, ${ }^{20}$ Neil Caporaso, ${ }^{20}$ Paul Brennan, ${ }^{2}$ Simone Benhamou, ${ }^{21}$ Veronique Benhaim-Luzon, ${ }^{2}$ Hans Kromhout, ${ }^{22}$ Roel Vermeulen, ${ }^{22}$ Susan Peters, ${ }^{22}$ Dario Consonni, ${ }^{23}$ Bas Bueno-de-Mesquita, ${ }^{24}$ Paolo Boffetta, ${ }^{25}$ Kurt Straif ${ }^{2}$ Karolinska Institutet, Stockholm, Sweden; ${ }^{2}$ IARC, Lyon, France; ${ }^{3} P$ PA, Bochum, Germany; ${ }^{4}$ University of Duisburg-Essen, Essen, Germany; ${ }^{5}$ Bremen Institute for Prevention Research and Social Medicine, Bremen, Germany; ${ }^{6}$ Deutsches Forschungszentrum fur Gesundheit und Umwelt, Neuherberg, Germany; ${ }^{7}$ University of Turin, Turin, Italy; ${ }^{8}$ Russian Cancer Research Centre, Moscow, Russia; ${ }^{9}$ Cancer Research Centre, University of Liverpool, Liverpool, UK; ${ }^{10}$ Nofer Institute, Lodz, Poland; ${ }^{11}$ National Institute of Environment Health, Budapest, Hungary; ${ }^{12}$ Cancer Center and Institute of Oncology, Warsaw, Poland; ${ }^{13}$ INSERM U 754 - IFR69, Villejuif, France; ${ }^{14}$ Regional Authority of Public Health, Banska Bystrica, Slovakia; ${ }^{15} / n$ stitute of Public Health, Bucharest, Romania; ${ }^{16}$ Institute of Hygiene and Epidemiology, Charles University, Prague, Czech Republic; ${ }^{17}$ Masaryk Memorial Cancer Institute, Brno, Czech Republic; ${ }^{18}$ Palacky University, Olomouc, Czech Republic; ${ }^{19}$ University of Montreal, Montreal, Canada; ${ }^{20} \mathrm{NCl}$, Bethesda, USA; ${ }^{21}$ INSERM U 946, Paris, France; ${ }^{22}$ Utrecht University, Utrecht, The Netherlands; ${ }^{23}$ Fondazione IRCCS Ca'Granda - Ospedale Maggiore Policlinico, Milan, Italy; ${ }^{24}$ NIVM, Bilthoven, The Netherlands; ${ }^{25}$ Mount Sinai School of Medicine, New York, USA 